\title{
Article \\ Pattern Formation upon Evaporation of Sessile Droplets of Polyelectrolyte/Surfactant Mixtures on Silicon Wafers
}

\author{
Lionel Perrin ${ }^{1,2, *}$, Andrew Akanno ${ }^{1}$, Eduardo Guzman 1,3 (), Francisco Ortega ${ }^{1,3}$ and Ramon G. Rubio $1,3, *$ (1) \\ 1 Departamento de Química Física, Facultad de Ciencias Químicas, Universidad Complutense, \\ Ciudad Universitaria s/n, 28040 Madrid, Spain; drewakanno@gmail.com (A.A.); \\ eduardogs@quim.ucm.es (E.G.); fortega@quim.ucm.es (F.O.) \\ 2 Institute Lumière Matière, Claude Bernard University Lyon 1, Bâtiment Alfred Kastler-4ème Etage Domaine \\ Scientifique de La Doua, 10 Rue Ada Byron, CEDEX, 69622 Villeurbanne, France \\ 3 Instituto Pluridisciplinar, Universidad Complutense, Paseo Juan XXIII 1, 28040 Madrid, Spain \\ * Correspondence: 1.perrin88@gmail.com (L.P.); rgrubio@quim.ucm.es (R.G.R.); Tel.: +34-3944123 (R.G.R.)
}

check for updates

Citation: Perrin, L.; Akanno, A.; Guzman, E.; Ortega, F.; Rubio, R.G. Pattern Formation upon Evaporation of Sessile Droplets of Polyelectrolyte/ Surfactant Mixtures on Silicon Wafers Int. J. Mol. Sci. 2021, 22, 7953. https://doi.org/10.3390/ ijms22157953

Academic Editors: Ana M. Mainar and José S. Urieta

Received: 14 June 2021

Accepted: 23 July 2021

Published: 26 July 2021

Publisher's Note: MDPI stays neutral with regard to jurisdictional claims in published maps and institutional affiliations.

Copyright: (c) 2021 by the authors. Licensee MDPI, Basel, Switzerland. This article is an open access article distributed under the terms and conditions of the Creative Commons Attribution (CC BY) license (https:// creativecommons.org/licenses/by/ $4.0 /)$.

\begin{abstract}
The formation of coffee-ring deposits upon evaporation of sessile droplets containing mixtures of poly(diallyldimethylammonium chloride) (PDADMAC) and two different anionic surfactants were studied. This process is driven by the Marangoni stresses resulting from the formation of surface-active polyelectrolyte-surfactant complexes in solution and the salt arising from the release of counterions. The morphologies of the deposits appear to be dependent on the surfactant concentration, independent of their chemical nature, and consist of a peripheral coffee ring composed of PDADMAC and PDADMAC-surfactant complexes, and a secondary region of dendrite-like structures of pure $\mathrm{NaCl}$ at the interior of the residue formed at the end of the evaporation. This is compatible with a hydrodynamic flow associated with the Marangoni stress from the apex of the drop to the three-phase contact line for those cases in which the concentration of the complexes dominates the surface tension, whereas it is reversed when most of the PDADMAC and the complexes have been deposited at the rim and the bulk contains mainly salt.
\end{abstract}

Keywords: evaporation; salt; surfactant; sessile droplet; patterning; Marangoni flow

\section{Introduction}

Contact angle, spreading, and evaporation phenomena are ubiquitous in nature and in technological processes, such as heat exchanges, ink-jet printing, pesticide applications, cosmetics and pharmacology, the food industry, etc. Despite the fact that the study of contact angle has attracted the attention of scientists since the 19th century, it still is an active field of research because new experimental and computational possibilities have revealed subtle details that had not been considered in the early times [1,2].

A simplified approach to the problem is sketched in Figure 1, where an axisymmetric fluid droplet is in equilibrium with two immiscible phases, e.g., solid and vapor or liquid and vapor, defining a three-phase contact line (TPCL). Either a thermodynamic or a mechanical approach to the problem can lead to the well-known Young equation

$$
\gamma_{\mathrm{sv}}-\gamma_{\mathrm{sl}}=\gamma_{\mathrm{lv}} \cos \theta
$$

where $\gamma_{\mathrm{sv}}, \gamma_{\mathrm{sl}}$, and $\gamma_{\mathrm{lv}}$ are the interfacial tensions of the solid/vapor, solid/liquid, and liid/vapor interfaces, respectively, and $\theta$ is the so-called equilibrium contact angle. 
a)

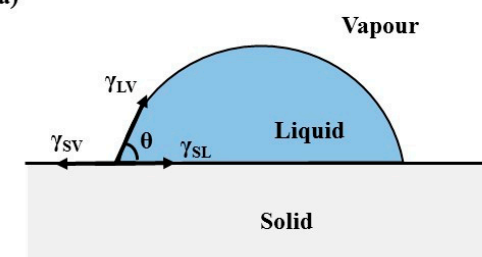

c)

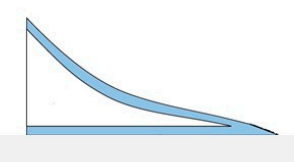

b)
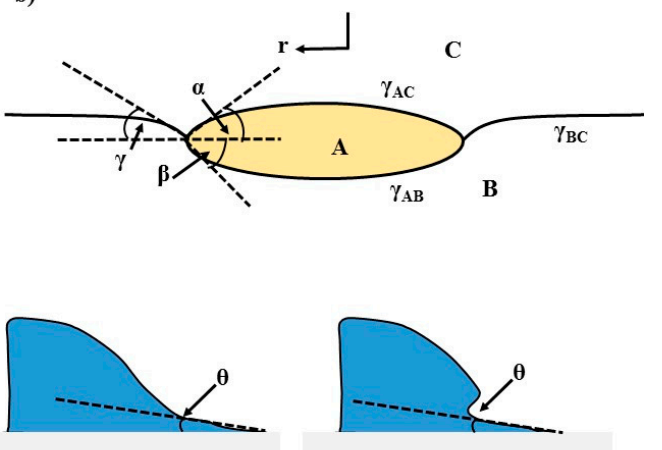

Figure 1. (a) Contact angle $(\theta)$ and interfacial tensions for an axisymmetric dropped onto a solid substrate. (b) Contact angles and interfacial tensions for an axisymmetric dropped onto a liquid surface (liquid lens), where A and B represent two liquid phases and C the third fluid (vapor or liquid). (c) Sketch of the liquid profile close to the three-phase contact line at a mesoscopic scale. (d) Sketch showing details of the real shape of the drop near the three-phase contact line of the liquid/solid/vapor interface at a mesoscopic scale.

It should be noted that the classical approach to the problem, though most frequently used for technological purposes, was developed for a macroscopic fluid droplet onto a perfectly smooth solid, and neglected the detailed physics dominating the behavior of the droplet near the TPCL. It must be considered that there are transitions between the bulk, where intermolecular potentials and hydrodynamics dominate the properties. Furthermore, the double layers of the liquid/vapor and solid/vapor interfaces are very far apart, to the region where they start to overlap, and Derjaguin forces start to dominate the behavior (see Figure 1c). Finally, a vapor layer adsorbed onto the solid, where statistical mechanics have to be considered. The continuity between the three regions above mentioned can lead to a complicated shape near the TPCL, as illustrated in Figure 1d. The inclusion of the Derjaguin forces is enough to explain the existence of the contact angle hysteresis $(\Delta \theta)$ usually observed in the experiments, even for very smooth surfaces. $\Delta \theta$ is defined as the difference between the advancing and the receding contact angles, $\Delta \theta=\theta_{\mathrm{a}}-\theta_{\mathrm{r}}$, and can take values of several degrees [3].

$\Delta \theta$ is crucial, both in theory and in practical applications, because when a drop is deposited on a substrate, two phenomena usually take place: spreading and evaporation. Both are non-equilibrium processes that are accompanied by hydrodynamic flows derived from the creation of Marangoni stresses [4]. If the substrates are not perfectly smooth, pinning and depinning phenomena complicate the situation, and even the loss of the circular shape of the TPCL can happen, as observed in patterned substrates [5].

In practical applications, mixtures instead of pure liquids are commonly used, which leads to time-dependent interfacial tensions associated with a differential adsorption of the components at the interfaces $[4,6]$. This leads to surface tension gradients which originate Marangoni stresses during the evaporation process. This emerges as a very interesting aspect from a technological perspective because many applications rely on the removal of the solvent excess from droplets of colloidal suspensions leading to deposits of solutes onto substrates whose surfaces are usually rough [7]. During evaporation, the droplet contact line can become pinned at a defect of the substrate surface for a significant period of time due to the balance between capillary-pressure and disjoining-pressure gradients. The pinning of the contact line accelerates droplet solidification, leading to particle deposition near the droplet edge which leads to the formation of a coffee-ring pattern [8-10]. Several recent reviews on the topic have been published [8,11,12]. In the case of mixtures with one or several non-volatile components, the advance of the evaporation pushes the mixtures beyond the saturation concentration of one or several components. This leads to precipitation and the so-called "coffee-ring" effect. It must be stressed that 
nature takes advantage of these interfacial phenomena, leading to striking behaviors such as those of superhydrophobic, superhydrophilic, and superomniphobic surfaces [13].

The absence of contact line pinning leads to the appearance of deposits with different morphologies, including concentric rings [14] and conical patterns [15]. In other studies, successive pinning and depinning of the contact line on the substrate surface has also been observed [16]. Among the most common patterns, ring-like shapes, bumps, multiple concentric rings, uniform deposits, or hexagonal arrays are included [17-21]. The suppression of the ring-shaped residue pattern is very useful for different technological applications, e.g., spray coating [22], microfabrication [23], bioassays [24,25], optoelectronic device manufacturing [26], DNA microchip fabrication [27,28], and production of solar energy absorption devices [29]. The patterns formed from drying biological fluids might be used for simple and rapid disease diagnosis [30]. Some empirical relationships between the patterns of dried droplets and different diseases have been established: carcinoma, viral hepatitis type B, Waldenstrom's disease, burn disease, tuberculosis, and leprosy [24,31,32]. It should be noted that even though the coffee-ring effect presents a high potential in many technological applications, it is deemed detrimental in many other fields, e.g., production of coatings [33] or detection of biomolecules using different spectroscopies [34]. The above discussion points out that the study of the behavior of a fluid drop onto a solid surface and in contact with its vapor is an intricate problem where equilibrium and non-equilibrium concepts cannot be separated. Therefore, the understanding of the physico-chemical aspects underlying the formation of the coffee-ring patterns upon the drying of droplets containing colloidal objects presents a paramount interest for both academia and industry.

This work describes the morphology and chemical composition of the coffee-ring-like deposits appearing on hydrophilic silicon dioxide surfaces upon the complete evaporation of a sessile droplets containing polyelectrolyte-surfactant mixtures. These mixtures are commonly included in shampoo-conditioner formulations, and hence their study presents key importance for the cosmetic industry, although the solid substrates used in this work are far from the complexity of real hair fibers, where the pinning effects will be stronger than in our substrates. It must be remarked that the performance of these formulations is related to the layer of polyelectrolyte-surfactant complexes adsorbed on the hair fibers after drying $[35,36]$. The optimization of the formulations makes it necessary to know the morphology of the deposit in order to minimize its friction coefficient. This work discusses the behavior of mixtures formed by poly(diallyldimethylammonium chloride) (PDADMAC) as polycation and two different anionic surfactants, sodium laureth sulfate (SLES) and sodium N-lauroyl-N-methyltaurate (SLMT). It should be noted that beyond the cosmetic industry, polyelectrolyte-surfactant mixtures present a broad interest in many other industries, e.g., detergency, tertiary oil recovery, production of dietary and pharmaceutical products, or wood pulping $[35,37,38]$. Therefore, the understanding of the pattern formation on surfaces upon the complete evaporation of aqueous solutions containing polyelectrolyte-mixtures is a very complex problem with a broad technological interest.

\section{Results}

The analysis of the deposits onto the hydrophilic silicon dioxide surfaces obtained after drying of droplets $(3-4 \mu \mathrm{L})$, at $25{ }^{\circ} \mathrm{C}$ and relative humidity $30 \%$, was performed on the bases of SEM images and the elemental analysis of the resulting patterns. It is worth noting that the mixtures of PDADMAC and the anionic surfactants (SLES or SLMT) were composed of positively charged PDADMAC-SLES or PDADMAC-SLMT complexes obtained upon the binding of the anionic surfactant to the polycation, which drives the release of the corresponding counterions $\left(\mathrm{Na}^{+}, \mathrm{Cl}^{-}\right)$resulting in a large entropy increase, as discussed in previous publications [39,40]. Further details on the characterization of the association and the interfacial properties related to the water/vapor and water/solid surfaces are discussed in our previous publications [40,41]. 


\subsection{SImages}

The SEM images of dried droplets for PDADMAC-SLES and PDADMAC-SLMT mixtures with different surfactant concentrations are shown in Figure 2. These images show the formation of patterns with similar morphology independently of the specific nature of the polyelectrolyte-surfactant mixture, and the variation of the structure of the deposits with surfactant concentration. The deposits cover the entire wetted area and their morphologies are consistent with the formation of coffee-ring-like patterns [42,43]. The morphologies are composed of a peripheral ring and a uniform distribution of crystals that starts from the rim and covers the entire pattern. The increase of the surfactant concentration leads to the emergence of a distinct crystalline structure that starts to accumulate in the center of the deposit. It must be stressed that the EDS spectra (discussed later) reveals that the entire surface of the pattern is covered by a thin layer of polyelectrolyte.
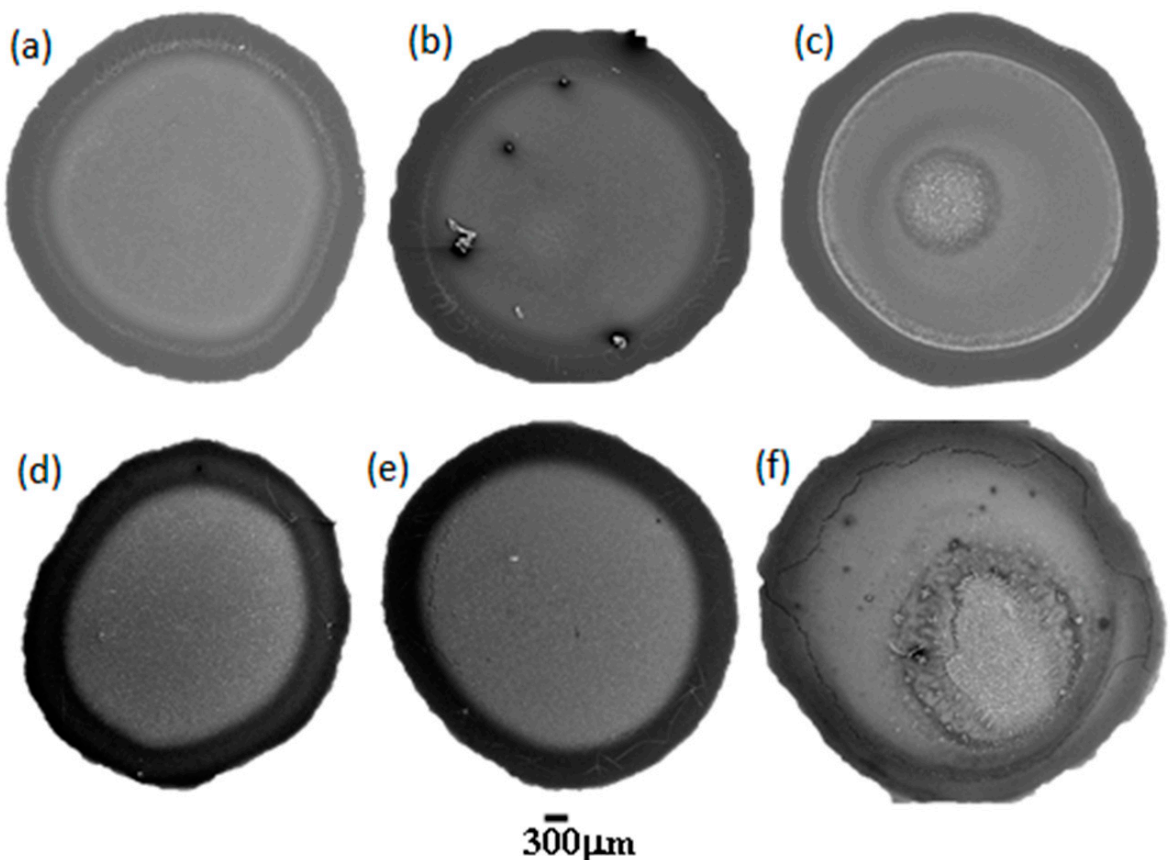

Figure 2. SEM images of dried droplets of PDADMAC-SLES mixtures with SLES concentrations of (a) $26 \mu \mathrm{M}$, (b) $0.26 \mathrm{mM}$, and (c) $2.6 \mathrm{mM}$, and of PDADMAC-SLMT mixtures with SLMT concentrations of (d) $2.9 \mu \mathrm{M}$, (e) $29 \mu \mathrm{M}$, and (f) $2.9 \mathrm{mM}$.

A detailed analysis of the patterns allows one to distinguish three different regions ("Region 1", "Region 2", and "Region 3") as is illustrated in Figure 3 for dried droplets of both mixtures. The combination of the SEM images corresponding to the deposits with their respective EDS spectra allows obtaining a better understanding of the distribution of the different species within the formed pattern (see Figure 4 for an example of the results obtained for one of the samples).

On the basis of the obtained results is possible to summarize the following characteristics for the obtained deposits:

i. Region 1 is the perimeter of the pattern, i.e., the coffee ring which appears in all of the deposits, in agreement with those reported for dried droplets of other complex mixtures $[43,44]$. The obtained results suggest that the thickness of the ring is independent of the surfactant concentration, which might be ascribed to the similar composition of the ring in all cases. This may be understood considering that the formation of the ring is associated with the presence of PDADMAC in the mixture which presents a constant concentration $(5 \mathrm{~g} / \mathrm{L})$. In addition, cracks, associated with the release of internal stresses which accumulate during the drying process, 
are observed in the ring areas which are more obvious when PDADMAC-SLMT deposits are considered.

ii. Region 2 is the secondary area of the deposits which is made up of crystal-like structures distributed uniformly within the droplet. These structures are $\mathrm{NaCl}$ crystals formed as a result of the release of counterions during the binding of the anionic surfactant to the polycation. The formation of this salt crystals agrees with the picture expected from the binding isotherm reported in our previous publication [40], with their deposition and growth being probably related to an anisotropy of surface tension between the substrate and the droplet mixture [45]. This may lead to a concentration gradient that favors the growth of the salt crystals, with such growth being commonly oriented towards the external polymeric ring. The results show that the salt crystals start to be formed from the edge of the pattern, appearing uniformly distributed close to the center of the droplets. It should be stressed the studied mixtures do not contain inorganic electrolytes, and hence the salt crystals can be only ascribed to the release of counterions occurring during the association between the PDADMAC and the surfactant. This contrasts with the salt crystal found in deposits obtained upon evaporation of other complex mixtures in which inorganic electrolytes were initially added $[45,46]$.

iii. Region 3 is located at the center of the obtained deposits, emerging as an accumulation of crystal structures.

It should be noted that the crystalline structures forming Region 3 are only observed at the highest surfactant concentration studied. The salt crystals are larger than those found in Region 2, which might be related to the direction of crystal growth observed in Region 2. This is plausible considering that the evaporation rate is similar for all the mixtures, and that the formation of these patterns emerges dependent on the drying rate $[46,47]$. Furthermore, the existence of Region 2 in all dried droplets indicates that the mechanism of pattern formation is similar, at least during the first part of the evaporation process, with independence of the surfactant concentration.

It should be stressed that the morphology of the deposits depends on whether $\mathrm{d} \gamma / \mathrm{d} c$, i.e., the slope of the surface tension isotherm corresponding to the adsorption of a surfaceactive molecule at the water/vapor interface, is positive or negative [48]. This was confirmed by $\mathrm{Hu}$ and Larsson [49] in their study about the evaporation of a polymer solution. They found the formation of volcano-like structures at the center of the deposit. Very recently, Efstratiou et al. [50] found the formation of a rim of evenly spaced salt crystals instead of a continuous rim after the evaporation of aqueous saline droplets. Furthermore, they also found that the type of salt, its initial concentration, and the hydrophilic or hydrophobic character of the substrate can drive the formation of salt deposits with very different morphologies, e.g., continuous rings, concentric rings, or discrete crystals, close to the ring or at the center of the deposit. It is worth mentioning that the evaporation of saline solutions is slowed down with respect to water because of the well-known decrease of the vapor pressure [51], which decreases the water vapor concentration far from the droplet surface. 


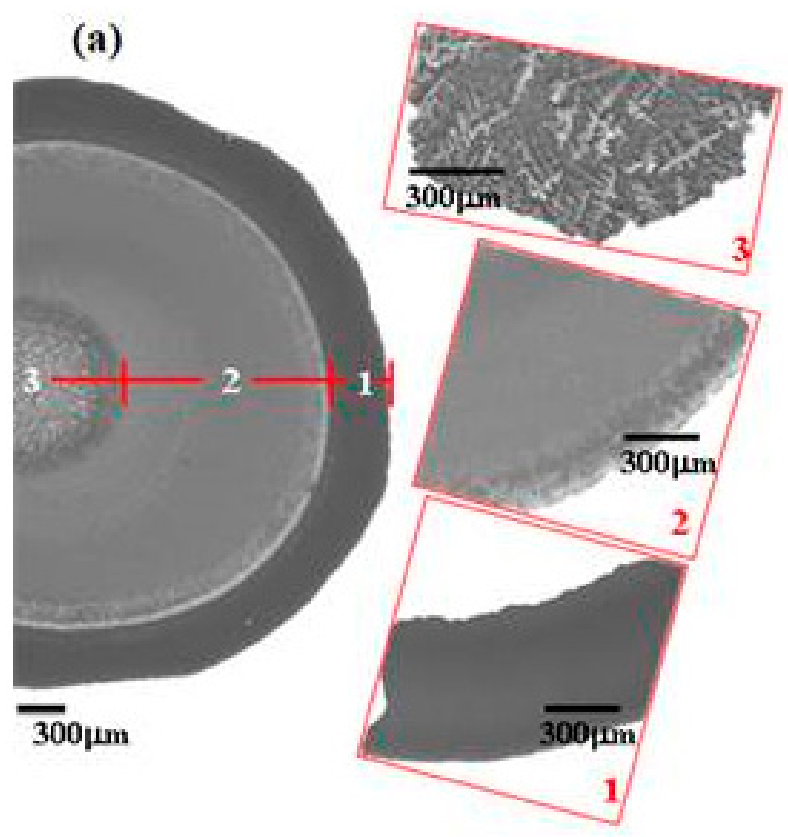

(b)

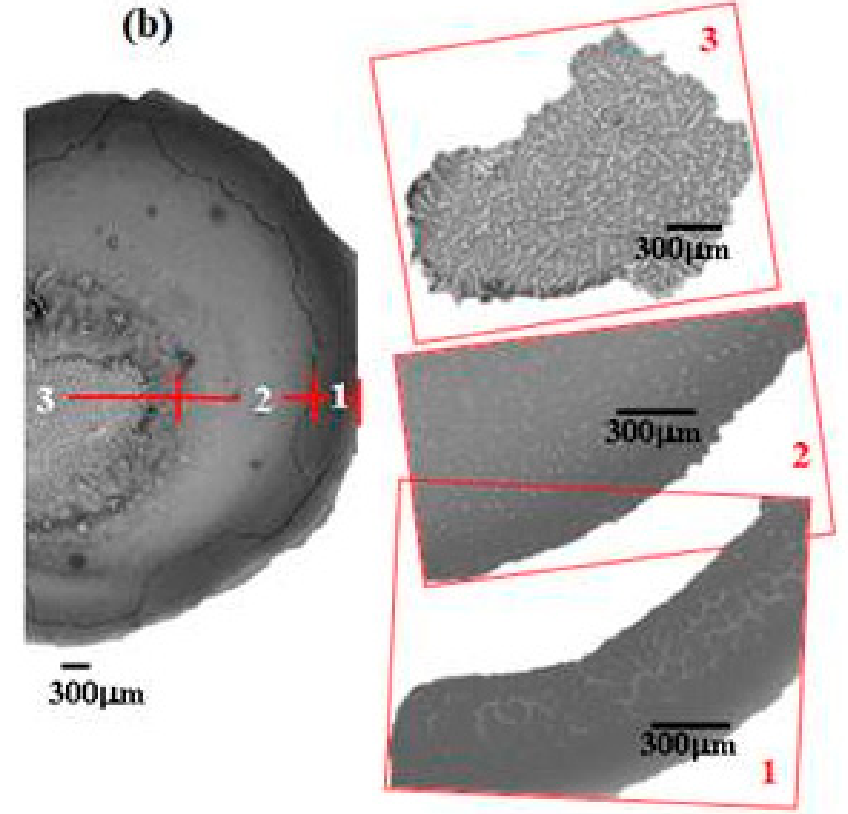

Figure 3. Dried droplets of PDADMAC-SLES (a) and PDADMAC-SLMT (b) mixtures, with surfactant concentrations of 2.6 and $2.9 \mathrm{mM}$, respectively, illustrating the three distinct regions. 


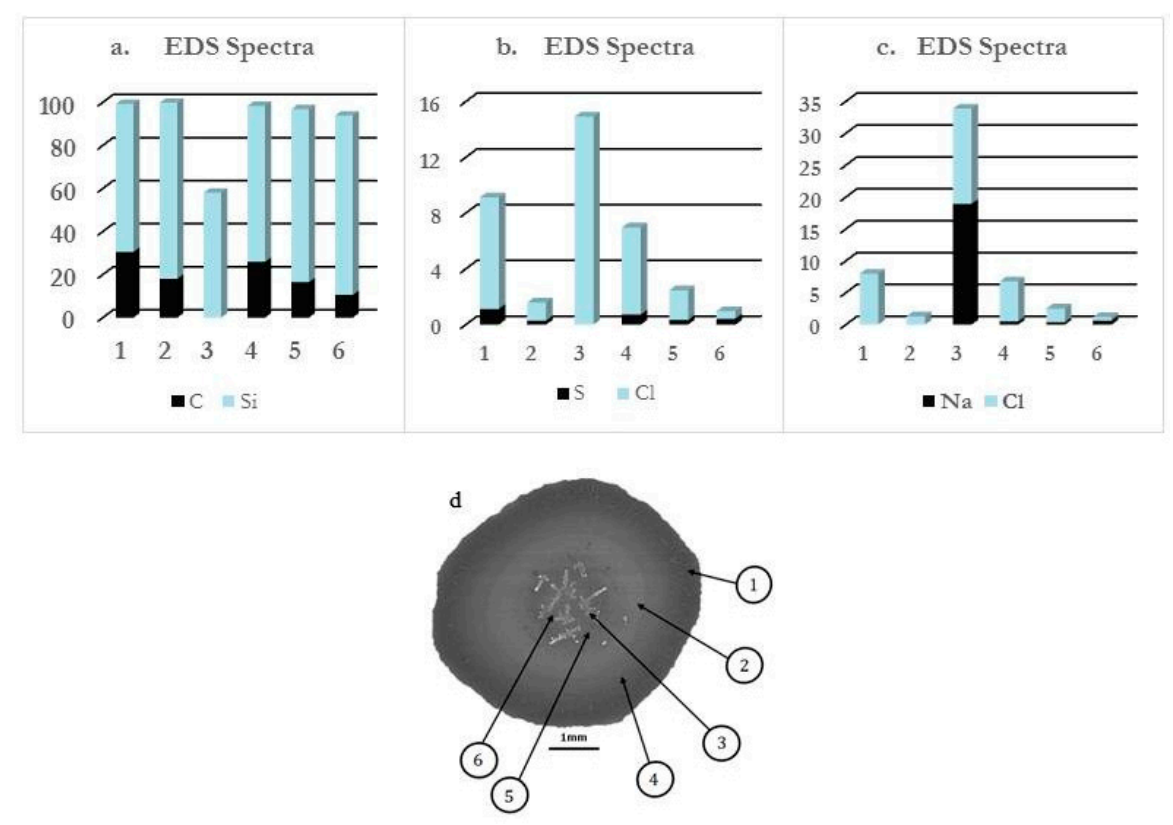

Figure 4. (a-c) EDS spectra showing the relative amounts of C, Si, S, Cl, and $\mathrm{Na}$ on 6 different spots of a deposit of PDADMAC-SLES mixture on a silica wafer. (d) SEM image of the deposit showing the 6 different spots. NB: spot 3 indicates the penetration of the EDS beam through the salt crystal.

To understand the accumulation of $\mathrm{NaCl}$ at the center of the deposit, the different sign of $\mathrm{d} \gamma / \mathrm{d} c$ and polyelectrolyte-surfactant mixtures must be taken into account. Thus, for salt solutions $d \gamma / d c>0$, whereas the sign is reversed $(d \gamma / d c<0)$ when polyelectrolyte-surfactant mixtures of intermediate concentrations are concerned, and assumes a quasi-null value $(\mathrm{d} \gamma / \mathrm{d} c \sim 0)$ value for mixtures with very low and very high surfactant. This picture is clear from the interfacial tension-surfactant concentration isotherms displayed in Figure 5 (further discussion on the specific dependences of the surface tension on the surfactant concentration for polyelectrolyte-surfactant solutions, and their differences with the isotherms for the surfactant solutions can be found in our previous publication [40]). The above-discussed differences in $\mathrm{d} \gamma / \mathrm{d} c$ provide a justification for the tendency of the Marangoni flow to move the polymer-surfactant complexes towards the three-phase contact line at the beginning of the drop evaporation when the salt concentration is relatively small. This is possible because the instantaneous volume of the droplets $V$ is not much smaller than their initial volume $V_{0}$ as was demonstrated in our previous paper [6]. Despite that the $\mathrm{d} \gamma / \mathrm{d} c$ is very small, thus leading to values of $\gamma \approx \gamma_{0}$ (with $\gamma_{0}$ being surface tension of water) for the initial salt solution, the salt concentration becomes very high during the latter stage of evaporation, so that $\gamma$ is several $\mathrm{mN} / \mathrm{m}^{-1}$ higher than $\gamma_{0}$. Furthermore, as was stated in our previous publication the $\gamma$ vs. surfactant concentration curves for the polymer-surfactant mixtures have the same shape as that of the surfactant solutions [40], and it is expected that $\mathrm{d} \gamma / \mathrm{d} c \approx 0$ for high surfactant concentrations as the evaporation proceeds. For the sake of example, for the lowest initial surfactant concentration $(2.6 \mu \mathrm{M})$, the surfactant concentration, $26 \mu \mathrm{M}$ at which $\gamma$ becomes constant is reached after an evaporation time $t=750 \mathrm{~s} \mathrm{[6].} \mathrm{At} \mathrm{this} \mathrm{time} \mathrm{the} \mathrm{only} \mathrm{contribution} \mathrm{to} \mathrm{the}$ Marangoni flow is that associated with the $\mathrm{NaCl}$ formed upon the binding of surfactant molecules to PDADMAC chains. The formation of salt deposits and the reversal of the coffee ring have been described in the last decades by other authors $[44,49,52,53]$. 


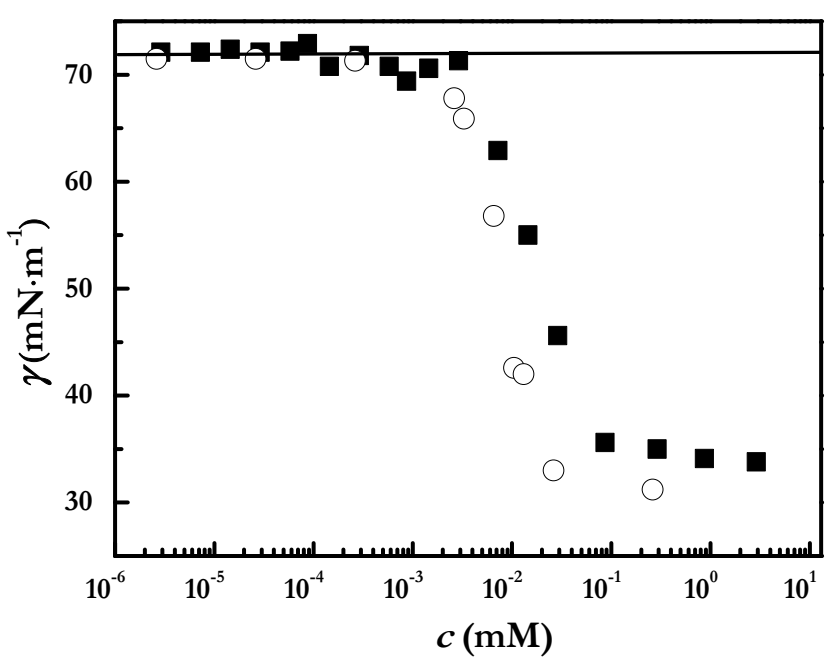

Figure 5. Surface tension isotherms as were obtained using a drop shape tensiometer for PDADMAC-surfactant solutions with a fixed polymer concentration of $5 \mathrm{~g} / \mathrm{L}$ : PDADMAC-SLMT (ם) and PDADMAC-SLES $(\bigcirc)$. The solid lines represent the surface tension of the pure Milli-Q water used in this work. Adapted from Akanno et al. [6], with permission under attribution license Creative Commons 4.0 (2021).

\subsection{Governing Mechanism for Crystal Formation and Crystal Size Variation with Surfactant Concentration}

The salt crystals formed on evaporation of the mixture droplets were observed to be dendrite-like structures as is shown in Figure 6. The physical mechanism governing selfforming dendrites from solutions have been studied extensively by Langer [54] on the basis of the Mullins-Sekerka instability concept, which accounts for the formation of dendrites with side branching. Mullins and Sekerka [55] explained that for a diffusion-controlled crystal growth, any instability in the system will affect the concentration gradients and favor the growth of the crystal.

The stability of a diffusion-controlled crystallization process of a solution on a solid surface is related to the gradient of the solute concentrations between the solid surface and the bulk solution and a capillary effect due to the surface tension at the solid-solution interface. Mullins and Sekerka [55] showed that the growth of the solute crystals on the solid surface is the result of some perturbations within the system, which are associated with a local solute concentration gradient. Furthermore, such a concentration gradient is also related to the solid-solution surface tension, and hence the variation of this quantity at the solid-liquid interface may enhance the importance of the instabilities.

In the case of evaporating sessile droplets of polyelectrolyte-surfactant mixtures, the existence of temperature gradients between the surface of the sessile droplet and the silicon wafer is an indication that the drying process is both thermal and diffusiondriven. Additionally, in the solid-liquid equilibrium, the salt concentration in the droplet is much greater than at the silicon wafer surface, thus $\mathrm{NaCl}$ molecules are constantly ejected to the solid surface in a similar way that latent heat is released from a melt during solidification [56], and the interfacial salt composition grows with time. On the other hand, the deposition of the positively charged complexes on the negatively charged silicon wafer surface may result in a charge inversion of the substrate surface [57]. The elemental analyses (EDS spectra displayed in Figure 4) of the patterns also reveal an increase in the height of the spectrum of silicon from the droplet rim to the central region which could mean that the thickness of the pattern formed on the silicon wafer decreases in the same direction as sketched in Figure 7. Therefore, there is the possibility that a gradient of charge distribution exists on the surface of the pattern from the more positively charged edge to the less positively charged central region. This will create a gradient of surface energy on the silicon wafer surface eventually leading to variations of the solid-liquid 
interfacial tension, which is likely the source of the interfacial instabilities in the case of the evaporating droplets of the mixtures in the current study.
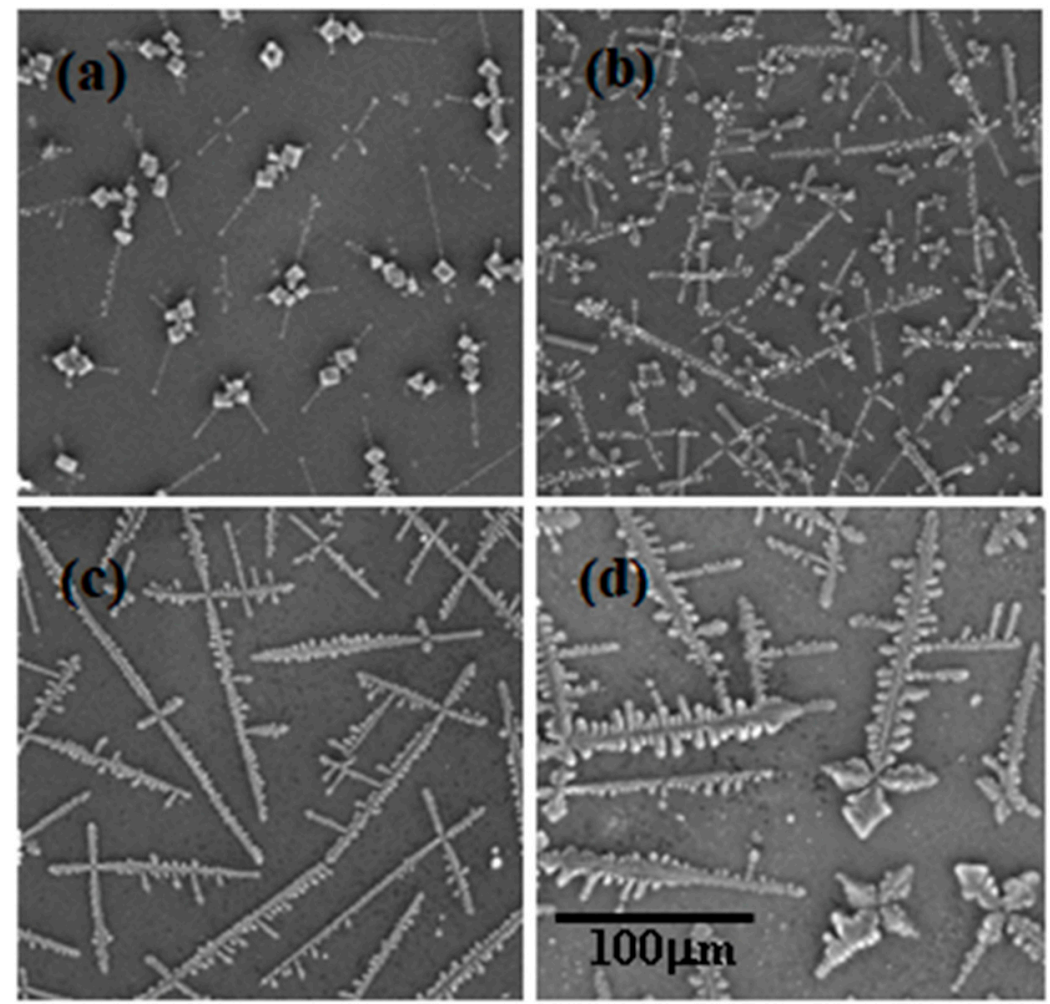

Figure 6. SEM images of dried droplets of PDADMAC-SLMT mixtures depicting the variation in $\mathrm{NaCl}$ crystal size with SLMT concentrations of: (a) $2.9 \mu \mathrm{M}$, (b) $29 \mu \mathrm{M}$, (c) $0.29 \mathrm{mM}$, and (d) $2.9 \mathrm{mM}$.

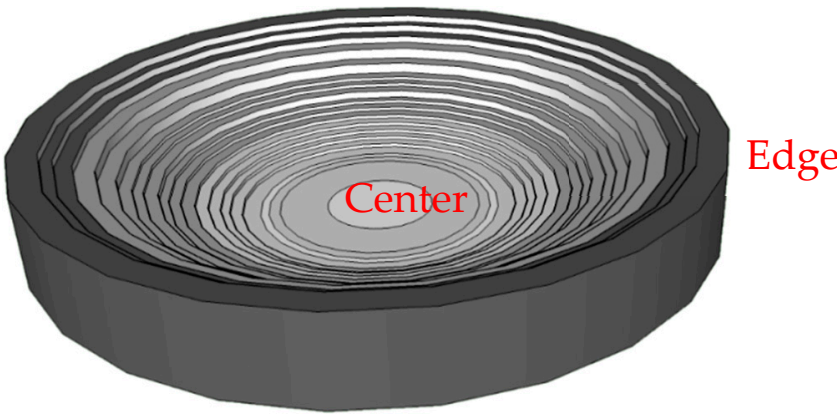

Figure 7. Idealized picture of the variation in thickness of the pattern formed by the evaporated droplet of the polyelectrolyte-surfactant mixture. The thickness of the deposit decreases from the edge (darker region) to the center (lighter region).

Mullins and Sekerka [55] stated that the instabilities would create a gradient in the interfacial salt concentration producing bulges and dimples on an initially growing spherical particle as is schematized in Figure 8. The increase of the perturbations in the system promotes the growth of the salt crystals observed in the patterns. Interestingly, these interfacial instabilities are unaffected by the solute concentration in the bulk solution [57], and therefore salt crystals were observed in the patterns formed for all the surfactant concentrations. Furthermore, the structures presented in the current study consist of a main branch with a length of hundreds of microns connected to shorter branches on the sides. Additionally, the side branches vary in size and appear to be equally spaced on the main branch. Furthermore, the size of these dendrites increases with the surfactant concentration, which can be linked to an increase in the concentration of the counterions 
in the mixture. It should be noted that the concentrations of $\mathrm{Na}^{+}$and $\mathrm{Cl}^{-}$in the solution are very far from the saturation concentration of aqueous $\mathrm{NaCl}$ solution $(6.15 \mathrm{M})$ [58], and hence it may be expected that only close to the complete evaporation of the droplets do well-defined dendritic-like crystals appear on the silicon wafer surface. This increase may be only apparent because, as was already mentioned, $\mathrm{Na}^{+}$and $\mathrm{Cl}^{-}$ions are also present in the rim of the deposit, although no quantitative analysis of the real salt concentration can be done with the present data. Moreover, it cannot be forgotten that when $\mathrm{NaCl}$ crystals start to appear, tiny amounts of surfactant and polymer may still be present in the solution. The high solubility of $\mathrm{NaCl}$ in water provides a justification for the increase of the size of the dendritic-like structures with the surfactant concentration. Thus, the higher the surfactant concentration, the easier the depletion of the $\mathrm{NaCl}$ from the aqueous solution, and hence the growth of dendritic-like structures appears favored.

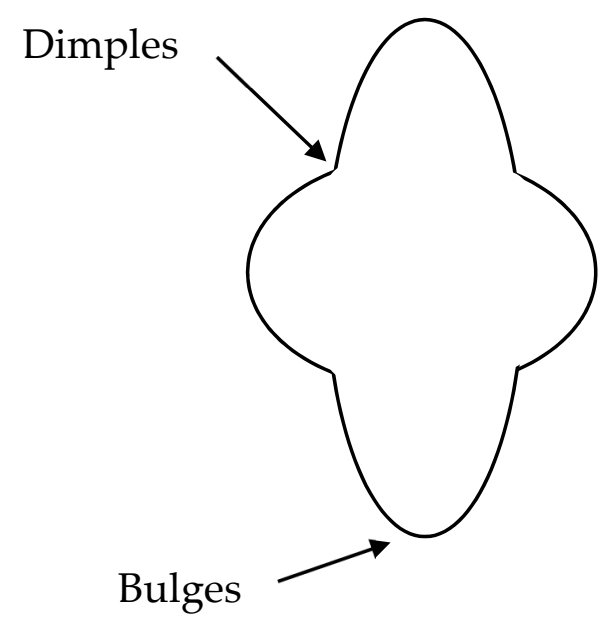

Figure 8. Idealized picture representing the perturbed state of an initially spherical particle under the influence of interfacial instabilities.

In addition to the presence of interfacial instabilities at the solid-liquid interface promoting the growth of crystal structures on the solid surface, Coriell and Sekerka [59] pointed out that the existence of anisotropy in the solid-liquid interfacial tension will cause these instabilities to be direction dependent. The crystals observed in the patterns formed upon the evaporation of droplets of PDADMAC-SLES and PDADMAC-SLMT mixtures were randomly distributed. This leads to well-defined directional growth which agrees with the existence of anisotropy in surface tension at the solid-liquid interface within the droplet of the mixture and the PDADMAC-modified substrate surface. Finally, it must be taken into account that, once crystallization starts, a local concentration gradient is created because in the immediate vicinity of the crystal surface the solution is supersaturated and at a very short distance it is saturated. Such gradient creates an associated surface tension gradient that establishes a jet-like flow towards the growing crystal edge (recall that when crystallization takes place, there is almost only salt in the solution, thus $\mathrm{d} \gamma / \mathrm{d} c>0$ ).

\section{Materials and Methods}

\subsection{Chemicals}

PDADMAC with a weight average molecular mass $M_{\mathrm{w}}$ in the 100-200 kDa range was purchased as a $200 \mathrm{~g} / \mathrm{L}$ aqueous solution from Sigma-Aldrich (St. Louis, MO, USA) and was used as received. The anionic surfactant SLES, with an average number of 2 oxyethylene, was supplied by Kao Chemical Europe S.L. (Barcelona, Spain) as an aqueous solution of $70 \mathrm{wt} \%$ of surfactant concentration. It was purified by lyophilization followed by recrystallization of the obtained powder using acetone for HPLC (Acros Organics, Morris Plains, NJ, USA) [60]. SLMT was synthesized and purified following the procedure described in our previous publication [39]. 
Ultrapure deionized water used for cleaning and solution preparation was obtained by a multicartridge purification system aquaMAX ${ }^{\mathrm{TM}}$-Ultra 370 Series (Young Lin Instrument, Co., Anyang, Korea). The water used had a resistivity higher than $18 \mathrm{M} \Omega \cdot \mathrm{cm}$, and a total organic content lower than $6 \mathrm{ppm}$.

\subsection{Preparation of the Polyelectrolyte-Surfactant Solutions}

Aqueous solutions of polyelectrolyte-surfactant mixtures, containing a fixed PDADMAC concentration of $5 \mathrm{~g} / \mathrm{L}$ and different surfactant concentrations in the range of $10^{-6}-5 \mathrm{mM}$ were used. The preparation of the aqueous mixtures was performed according to the procedure reported in our previous work [39]. This procedure can be briefly summarized in the following steps: (i) the required amount of the aqueous commercial solution of PDADMAC for obtaining final mixtures with a polymer concentration of $5 \mathrm{~g} / \mathrm{L}$ was weighed and poured into a flask; (ii) surfactant was added to the flask containing the polyelectrolyte. For this purpose, surfactant solutions $(\mathrm{pH} \sim 5.6)$ with concentrations one order of magnitude higher than the surfactant concentration required in the final mixture were weighted and added to the flasks containing PDADMAC. (iii) The mixture was diluted with an acetic acid solution of $\mathrm{pH} \sim 5.6$ to the final bulk composition. During the mixture preparation, there was no delay between the addition of the different components. The final mixtures were homogenized by mild stirring at $1000 \mathrm{rpm}$ using a magnetic stirrer for $1 \mathrm{~h}$ at room temperature, and then they were left to age for 1 week prior to use. This aging period was used to ensure that no turbidity appeared in the mixtures and that the transparency of the mixtures after preparation was maintained [39]. The above procedure has been shown to demonstrate a good level of reproducibility for obtaining kinetically arrested states after mixing the PDADMAC and surfactant [39].

The $\mathrm{pH}$ of all the solutions was fixed at 5.6 using glacial acetic acid (purity $>99 \%$ ). It should be noted that the $\mathrm{pH}$, the PDADMAC concentration, and the use of acetic acid for fixing the $\mathrm{pH}$ were not arbitrary choices. Such conditions were adopted to mimic the characteristics of hair-conditioning formulations used for reducing the bleaching of hair fibers under application conditions [61,62].

\subsection{Preparation of the Silicon Wafer Surface}

Silicon wafers 1 inch in diameter were supplied by Siltronix (Archamps, France) and were cleaned, just before their use, by immersion in piranha solution (mixture of sulfuric acid and hydrogen peroxide in a volume ratio 7:3) for $30 \mathrm{~min}$, followed by rinsing with Milli-Q water and absolute ethanol. Afterwards, the wafers were dried under a nitrogen stream. This procedure resulted in the formation of a homogeneous layer of hydrophilic silicon dioxide on the wafer surface, with a thickness in the range $2-3 \mathrm{~nm}$ as was determined by ellipsometry.

\subsection{Scanning Electron Microscope}

The morphologies of the patterns formed on the surfaces after evaporation of the mixtures were analyzed using scanning electron microscopy (SEM). Two different Scanning electron microscopes, with magnification up to 5000× , from JEOL (models JSM 6400 and JSM 6335F-Akhisima, Japan) were used for studying the morphologies and structures of the obtained deposits. The JSM 6400 operates with a beam voltage of $25 \mathrm{kV}$ and presents image resolution of up to $10 \mathrm{~nm}$, whereas the JSM 6335F operates at a lower beam voltage $(15 \mathrm{kV})$, enhancing the resolution up to $1.5 \mathrm{~nm}$. Furthermore, the latter is coupled to an energy-dispersive X-ray spectrometer (EDS) allowing us to perform the elemental analysis of the deposits.

\subsection{Surface Tension Measurements}

The dependences of the equilibrium interfacial tension $\gamma$ on the surfactant concentration upon adsorption of polyelectrolyte-surfactant aqueous solutions to the water/vapor interface was followed using a home-made profile analysis tensiometer in pendant drop 
configuration (for further details see reference [39]). The adsorption at the water/vapor interface was measured until a steady state was reached, i.e., changes of surface tension smaller than $0.1 \mathrm{mN} \cdot \mathrm{m}^{-1}$ during $30 \mathrm{~min}$. Special care was taken to minimize the evaporation effects during these experiments.

\section{Conclusions}

The patterns formed on the silicon wafers upon the complete evaporation of sessile droplets of polyelectrolyte-surfactant mixtures present two well-differentiated regions, with the appearance of a third one for mixtures with high surfactant concentration. The formation of patterns on the substrate leads to a pinning of the contact line to the substrate as result of the deposition of PDADMAC at the periphery of the droplet, which is strongly favored due to the electrostatic interactions at the PDADMAC-surface. The relatively constant size of the ring is an indication that the coffee ring is mainly due to the PDADMAC in the mixture and independent of the size of the PDADMAC-surfactant complexes formed in the mixture. Furthermore, the appearance of cracks around the coffee ring, associated with the release of internal stress that accumulates during evaporation, is observed. Capillary and solutal Marangoni flows drive the deposition of PDADMAC molecules and PDADMAC-surfactant complexes at the periphery of the deposits as the evaporation proceeds, with the resulting pattern depending on the surfactant concentration, even though no influence of the nature of the surfactant was found. The peripheral zone is followed by a secondary region composed of dendrite-like structures of $\mathrm{NaCl}$ uniformly distributed in the interior of the pattern. These $\mathrm{NaCl}$ crystals are the result of precipitation of the counterions released during the binding of the surfactant to the polyelectrolyte chains in a salt-free mixture, with their size increasing with surfactant concentration. The deposition of salt crystals in salt-free solutions should be considered a new physico-chemical scenario because previous studies dealing with salt crystal formation after dying were concerned to systems in which inorganic electrolytes were initially contained in the solution.

The formation of the dendrite-like structures may result from the instabilities produced due to surface tension anisotropies which are promoted throughout the interior of the droplet of the mixtures. This instability appears after PDADMAC-surfactant complex deposition. Furthermore, the motion of the counterions towards the center of the drop leads to a uniform distribution of the salt crystals in the interior of the ring. At the highest surfactant concentration, the excess concentration of counterions produced in the mixtures was observed to precipitate and accumulate around the center of the droplet forming a third region of large dendrite structures.

The final patterns result from two different Marangoni flows which present different circulation. This may be rationalized in terms of the different $d \gamma / d c$ existing for polyelectrolyte-surfactant complexes and for counterions. To the best of our knowledge, this work reports the first evidence of such type of phenomena. Furthermore, another interesting aspect of the crystal formation in the current study is that they are self-growing and only appear in the presence of the polyelectrolyte. Presumably, the accumulation of PDADMAC-surfactant complexes at the contact line provides the suitable environment for crystallization of the salt as no crystal structure was observed with a salt solution of the same concentration as the counterions in the mixtures.

The understanding of the here-contained aspects presents a critical impact in the selection of materials for coating applications as these salt crystals might influence the desired properties e.g., strongly modify the spreading properties on the coated surface or its friction coefficient.

Author Contributions: Conceptualization, L.P. and R.G.R.; methodology, A.A. and L.P.; software, A.A., L.P. and E.G.; validation, L.P. and R.G.R.; formal analysis, L.P.; investigation, A.A., L.P., E.G., F.O. and R.G.R.; resources, F.O. and R.G.R.; data curation, L.P. and R.G.R.; writing-original draft preparation, A.A., L.P., E.G. and R.G.R.; writing—review and editing, L.P., E.G., F.O. and R.G.R.; visualization, E.G.; supervision, R.G.R.; project administration, R.G.R.; funding acquisition, E.G., F.O. and R.G.R. All authors have read and agreed to the published version of the manuscript. 
Funding: This work was funded in part by MICINN (Spain) under grant PID2019-106557GB-C21, by Banco Santander-Universidad Complutense grant PR87/19-22513 (Spain) and by E.U. on the framework of the European Innovative Training Network-Marie Sklodowska-Curie Action NanoPaint (grant agreement 955612).

Institutional Review Board Statement: Not applicable.

Informed Consent Statement: Not applicable.

Data Availability Statement: Data are available upon request.

Acknowledgments: Authors thank the Centro the Espectroscopía y Correlación of Complutense University of Madrid and Centro de Nacional de Microscopía Electrónica for the use of some of its facilities.

Conflicts of Interest: The authors declare no conflict of interest. The funders had no role in the design of the study; in the collection, analyses, or interpretation of data; in the writing of the manuscript, or in the decision to publish the results.

\section{Abbreviations}

$\Gamma \quad$ equilibrium interfacial tension

$\gamma_{0} \quad$ surface tension of the water

$\gamma_{\mathrm{sv}} \quad$ interfacial tension of the solid/vapor interface

$\gamma_{\mathrm{sl}} \quad$ interfacial tension of the solid/liquid interface

$\gamma_{\mathrm{lv}} \quad$ interfacial tension of the liquid/vapor interface

$\Theta \quad$ equilibrium contact angle

$\theta_{\mathrm{a}} \quad$ advancing contact angle

$\theta_{\mathrm{r}} \quad$ receding contact angle

$\Delta \theta \quad$ contact angle hysteresis

C surfactant concentration

EDS energy-dispersive $\mathrm{X}$-ray spectrometer

$M_{\mathrm{W}} \quad$ weight average molecular mass

PDADMAC poly(diallyldimethylammonium chloride)

SEM scanning electron microscopy

SLES Sodium laureth sulfate

SLMT sodium N-lauroyl-N-methyltaurate

T time

TPCL three-phase contact line

$V \quad$ instantaneous volume of the droplet

$V_{0} \quad$ initial volume of the droplet

\section{References}

1. Starov, V.M.; Velarde, M.G. Wetting and Spreading Dynamics; CRC Press: Boca Raton, FL, USA, 2019.

2. Butt, H.-J.; Kappl, M. Surface and Interfacial Forces; Wiley-VCH: Berlin, Germany, 2010.

3. Lyklema, J. (Ed.) Fundamentals of Interface and Colloid Science: Solid-Liquid Interfaces; Academic Press: San Diego, CA, USA, 1995; Volume 2.

4. Semenov, S.; Trybala, A.; Rubio, R.G.; Kovalchuk, N.; Starov, V.; Velarde, M.G. Simultaneous spreading and evaporation: Recent developments. Adv. Colloid Interface Sci. 2014, 206, 382-398. [CrossRef]

5. Huang, Q.; Zhang, Z.; Liu, Z.; Zhang, F.; Cheng, G.; Ding, J. Pinning effect in droplet self-driving and its reduction mechanism by monolayer graphene. Appl. Surf. Sci. 2021, 542, 148666. [CrossRef]

6. Akanno, A.; Perrin, L.; Guzmán, E.; Llamas, S.; Starov, V.M.; Ortega, F.; Rubio, R.G.; Velarde, M.G. Evaporation of Sessile Droplets of Polyelectrolyte/Surfactant Mixtures on Silicon Wafers. Colloids Interfaces 2021, 5, 12. [CrossRef]

7. Pham, T.; Kumar, S. Drying of Droplets of Colloidal Suspensions on Rough Substrates. Langmuir 2017, 33, 10061-10076. [CrossRef]

8. Larson, R.G. Transport and deposition patterns in drying sessile droplets. AIChE J. 2014, 60, 1538-1571. [CrossRef]

9. Parsa, M.; Harmand, S.; Sefiane, K. Mechanisms of pattern formation from dried sessile drops. Adv. Colloid Interface Sci. 2018, 254, 22-47. [CrossRef] [PubMed]

10. Still, T.; Yunker, P.J.; Yodh, A.G. Surfactant-Induced Marangoni Eddies Alter the Coffee-Rings of Evaporating Colloidal Drops. Langmuir 2012, 28, 4984-4988. [CrossRef]

11. Al-Milaji, K.N.; Zhao, H. New Perspective of Mitigating the Coffee-Ring Effect: Interfacial Assembly. J. Phys. Chem. C 2019, 123, 12029-12041. [CrossRef] 
12. Mampallil, D.; Eral, H.B. A review on suppression and utilization of the coffee-ring effect. Adv. Colloid Interface Sci. 2018, 252, 38-54. [CrossRef]

13. Guo, H.; Fuchs, P.; Casdorff, K.; Michen, B.; Chanana, M.; Hagendorfer, H.; Romanyuk, Y.E.; Burgert, I. Bio-Inspired Superhydrophobic and Omniphobic Wood Surfaces. Adv. Mat. Interfaces 2017, 4, 1600289. [CrossRef]

14. Mondal, R.; Basavaraj, M.G. Influence of the drying configuration on the patterning of ellipsoids-Concentric rings and concentric cracks. Phys. Chem. Chem. Phys. 2019, 21, 20045-20054. [CrossRef] [PubMed]

15. Willmer, D.; Baldwin, K.A.; Kwartnik, C.; Fairhurst, D.J. Growth of solid conical structures during multistage drying of sessile poly(ethylene oxide) droplets. Phys. Chem. Chem. Phys. 2010, 12, 3998-4004. [CrossRef]

16. Maheshwari, S.; Zhang, L.; Zhu, Y.; Chang, H.-C. Coupling between Precipitation and Contact-Line Dynamics: Multiring Stains and Stick-Slip Motion. Phys. Rev. Lett. 2008, 100, 044503. [CrossRef]

17. Baldwin, K.A.; Roest, S.; Fairhurst, D.J.; Sefiane, K.; Shanahan, M.E.R. Monolith formation and ring-stain suppression in low-pressure evaporation of poly(ethylene oxide) droplets. J. Fluid Mech. 2012, 695, 321-329. [CrossRef]

18. Orejon, D.; Sefiane, K.; Shanahan, M.E.R. Stick-Slip of Evaporating Droplets: Substrate Hydrophobicity and Nanoparticle Concentration. Langmuir 2011, 27, 12834-12843. [CrossRef]

19. Bhardwaj, R.; Fang, X.; Attinger, D. Pattern formation during the evaporation of a colloidal nanoliter drop: A numerical and experimental study. New J. Phys. 2009, 11, 075020. [CrossRef]

20. Keseroğlu, K.; Çulha, M. Assembly of nanoparticles at the contact line of a drying droplet under the influence of a dipped tip. $J$. Colloid Interface Sci. 2011, 360, 8-14. [CrossRef]

21. Maillard, M.; Motte, L.; Pileni, M.-P. Rings and Hexagons Made of Nanocrystals. Adv. Mat. 2001, 13, 200-204. [CrossRef]

22. Majumder, M.; Rendall, C.; Li, M.; Behabtu, N.; Eukel, J.A.; Hauge, R.H.; Schmidt, H.K.; Pasquali, M. Insights into the physics of spray coating of SWNT films. Chem. Eng. Sci. 2010, 65, 2000-2008. [CrossRef]

23. Chung, S.; Ul Karim, M.A.; Spencer, M.; Kwon, H.-J.; Grigoropoulos, C.P.; Alon, E.; Subramanian, V. Exploitation of the coffee-ring effect to realize mechanically enhanced inkjet-printed microelectromechanical relays with U-bar-shaped cantilevers. Appl. Phys. Lett. 2014, 105, 261901. [CrossRef]

24. Sefiane, K. On the Formation of Regular Patterns from Drying Droplets and Their Potential Use for Bio-Medical Applications. J. Bionic Eng. 2010, 7, S82-S93. [CrossRef]

25. Wen, J.T.; Ho, C.-M.; Lillehoj, P.B. Coffee Ring Aptasensor for Rapid Protein Detection. Langmuir 2013, 29, 8440-8446. [CrossRef]

26. Kong, Y.L.; Tamargo, I.A.; Kim, H.; Johnson, B.N.; Gupta, M.K.; Koh, T.-W.; Chin, H.-A.; Steingart, D.A.; Rand, B.P.; McAlpine, M.C. 3D Printed Quantum Dot Light-Emitting Diodes. Nano Lett. 2014, 14, 7017-7023. [CrossRef]

27. Wu, J.; Wang, R.; Yu, H.; Li, G.; Xu, K.; Tien, N.C.; Roberts, R.C.; Li, D. Inkjet-printed microelectrodes on PDMS as biosensors for functionalized microfluidic systems. Lab Chip 2015, 15, 690-695. [CrossRef]

28. Fang, X.; Li, B.; Petersen, E.; Seo, Y.-S.; Samuilov, V.A.; Chen, Y.; Sokolov, J.C.; Shew, C.-Y.; Rafailovich, M.H. Drying of DNA Droplets. Langmuir 2006, 22, 6308-6312. [CrossRef]

29. Jin, H.; Qian, J.; Zhou, L.; Yuan, J.; Huang, H.; Wang, Y.; Tang, W.M.; Chan, H.L.W. Suppressing the Coffee-Ring Effect in Semitransparent $\mathrm{MnO}_{2}$ Film for a High-Performance Solar-Powered Energy Storage Window. ACS Appl. Mat. Interfaces 2016, 8, 9088-9096. [CrossRef]

30. Carreón, Y.J.P.; Ríos-Ramírez, M.; Moctezuma, R.E.; González-Gutiérrez, J. Texture analysis of protein deposits produced by droplet evaporation. Sci. Rep. 2018, 8, 9580. [CrossRef]

31. Yakhno, T.A.; Yakhno, V.G.; Sanin, A.G.; Sanina, O.A.; Pelyushenko, A.S.; Egorova, N.A.; Terentiev, I.G.; Smetanina, S.V.; Korochkina, O.V.; Yashukova, E.V. The informative-capacity phenomenon of drying drops. IEEE Eng. Med. Biol. Mag. 2005, 24, 96-104. [CrossRef]

32. Rapis, E. A change in the physical state of a nonequilibrium blood plasma protein film in patients with carcinoma. Tech. Phys. 2002, 47, 510-512. [CrossRef]

33. Layani, M.; Gruchko, M.; Milo, O.; Balberg, I.; Azulay, D.; Magdassi, S. Transparent Conductive Coatings by Printing Coffee Ring Arrays Obtained at Room Temperature. ACS Nano 2009, 3, 3537-3542. [CrossRef]

34. Marsico, A.L.M.; Duncan, B.; Landis, R.F.; Tonga, G.Y.; Rotello, V.M.; Vachet, R.W. Enhanced Laser Desorption/Ionization Mass Spectrometric Detection of Biomolecules Using Gold Nanoparticles, Matrix, and the Coffee Ring Effect. Anal. Chem. 2017, 89, 3009-3014. [CrossRef]

35. Llamas, S.; Guzmán, E.; Ortega, F.; Baghdadli, N.; Cazeneuve, C.; Rubio, R.G.; Luengo, G.S. Adsorption of polyelectrolytes and polyelectrolytes-surfactant mixtures at surfaces: A physico-chemical approach to a cosmetic challenge. Adv. Colloid Interface Sci. 2015, 222, 461-487. [CrossRef] [PubMed]

36. Fernández-Peña, L.; Guzmán, E.; Leonforte, F.; Serrano-Pueyo, A.; Regulski, K.; Tournier-Couturier, L.; Ortega, F.; Rubio, R.G.; Luengo, G.S. Effect of molecular structure of eco-friendly glycolipid biosurfactants on the adsorption of hair-care conditioning polymers. Colloids Surf. B 2020, 185, 110578. [CrossRef]

37. Lindman, B.; Antunes, F.; Aidarova, S.; Miguel, M.; Nylander, T. Polyelectrolyte-surfactant association-From fundamentals to applications. Colloid J. 2014, 76, 585-594. [CrossRef]

38. Fernández-Peña, L.; Guzmán, E. Physicochemical Aspects of the Performance of Hair-Conditioning Formulations. Cosmetics 2020, 7, 26. [CrossRef] 
39. Llamas, S.; Fernández-Peña, L.; Akanno, A.; Guzmán, E.; Ortega, V.; Ortega, F.; Csaky, A.G.; Campbell, R.A.; Rubio, R.G. Towards understanding the behavior of polyelectrolyte-surfactant mixtures at the water/vapor interface closer to technologically-relevant conditions. Phys. Chem. Chem. Phys. 2018, 20, 1395-1407. [CrossRef]

40. Guzmán, E.; Fernández-Peña, L.; Akanno, A.; Llamas, S.; Ortega, F.; Rubio, G.R. Two Different Scenarios for the Equilibration of Polycation-Anionic Solutions at Water-Vapor Interfaces. Coatings 2019, 9, 438. [CrossRef]

41. Llamas, S.; Guzmán, E.; Baghdadli, N.; Ortega, F.; Cazeneuve, C.; Rubio, R.G.; Luengo, G.S. Adsorption of poly (diallyldimethylammonium chloride)—Sodium methyl-cocoyl-taurate complexes onto solid surfaces. Colloids Surf. A 2016, 505, 150-157. [CrossRef]

42. Senses, E.; Black, M.; Cunningham, T.; Sukhishvili, S.A.; Akcora, P. Spatial ordering of colloids in a drying aqueous polymer droplet. Langmuir 2013, 29, 2588-2594. [CrossRef]

43. Tarasevich, Y.Y.; Pravoslavnova, D. Drying of a multicomponent solution drop on a solid substrate: Qualitative analysis. Tech. Phys. 2007, 52, 159-163. [CrossRef]

44. Gorr, H.M.; Zueger, J.M.; McAdams, D.R.; Barnard, J.A. Salt-induced pattern formation in evaporating droplets of lysozyme solutions. Colloids Surf. B 2013, 103, 59-66. [CrossRef] [PubMed]

45. Kaya, D.; Belyi, V.; Muthukumar, M. Pattern formation in drying droplets of polyelectrolyte and salt. J. Chem. Phys. 2010, 133, 114905. [CrossRef]

46. Chen, G.; Mohamed, J.G. Complex protein patterns formation via salt-induced self-assembly and droplet evaporation. Eur. Phys. J. E 2010, 33, 19-26. [CrossRef]

47. Choudhury, M.D.; Jana, S.; Dutta, S.; Tarafdar, S. Pattern formation in a drying drop of starch gel with sodium chloride. Colloids Surf. A 2013, 432, 110-118. [CrossRef]

48. Poulard, C.; Damman, P. Control of spreading and drying of a polymer solution from Marangoni flows. Eur. Phys. Lett. 2007, 80, 64001. [CrossRef]

49. Hu, H.; Larson, R.G. Marangoni Effect Reverses Coffee-Ring Depositions. J. Phys. Chem. B 2006, 110, 7090-7094. [CrossRef] [PubMed]

50. Efstratiou, M.; Christy, J.; Sefiane, K. Crystallization-Driven Flows within Evaporating Aqueous Saline Droplets. Langmuir 2020, 36, 4995-5002. [CrossRef]

51. Koga, Y. Solution Thermodynamics and Its Application to Aqueous Solutions; Elsevier: Amsterdam, The Netherlands, 2017.

52. Weon, B.M.; Je, J.H. Capillary force repels coffee-ring effect. Phys. Rev. E 2010, 82, 015305. [CrossRef] [PubMed]

53. Arangalage, M.; Li, X.; Lequeux, F.; Talini, L. Dual Marangoni effects and detection of traces of surfactants. Soft Matter 2018, 14, 3378-3386. [CrossRef] [PubMed]

54. Langer, J.S. Instabilities and pattern formation in crystal growth. Rev. Mod. Phys. 1980, 52, 1-28. [CrossRef]

55. Mullins, W.W.; Sekerka, R.F. Morphological stability of a particle growing by diffusion or heat flow. J. Appl. Phys. 1963, 34, 323-329. [CrossRef]

56. Fteiti, M.; Alaidrous, A. Latent Heat Storage during Melting and Solidification of a Phase Change Material (PCM) Embedded with a Porous Matrix of High Thermal Conductivity. Int. J. Energy Eng. 2020, 10, 1-9. [CrossRef]

57. Guzmán, E.; Ritacco, H.; Rubio, J.E.; Rubio, R.G.; Ortega, F. Salt-induced changes in the growth of polyelectrolyte layers of poly (diallyl-dimethylammonium chloride) and poly (4-styrene sulfonate of sodium). Soft Matter 2009, 5, 2130-2142. [CrossRef]

58. Pinho, S.P.; Macedo, E.A. Solubility of $\mathrm{NaCl}, \mathrm{NaBr}$, and $\mathrm{KCl}$ in Water, Methanol, Ethanol, and Their Mixed Solvents. J. Chem. Eng. Data 2005, 50, 29-32. [CrossRef]

59. Coriell, S.R.; Sekerka, R.F. The effect of the anisotropy of surface tension and interface kinetics on morphological stability. J. Cryst. Growth 1976, 34, 157-163. [CrossRef]

60. Friedrich, L.C.; Silva, V.O.; Moreira, P.F., Jr.; Tcacenco, C.M.; Quina, F.H. Time-resolved fluorescence quenching studies of sodium lauryl ether sulfate micelles. J. Braz. Chem. Soc. 2013, 24, 241-245. [CrossRef]

61. Gavazzoni Dias, M.F. Hair cosmetics: An overview. Int. J. Tribol. 2015, 7, 2-15. [CrossRef]

62. Wege, H.; Holgado-Terriza, J.A.; Cabrerizo-Vílchez, M. Development of a constant surface pressure penetration Langmuir balance based on axisymmetric drop shape analysis. J. Colloid Interface Sci. 2002, 249, 263-273. [CrossRef] 